\title{
Off-Line Processing: Reciprocal Interactions between Declarative and Procedural Memories
}

\author{
Rachel M. Brown and Edwin M. Robertson \\ Center for Noninvasive Brain Stimulation, Beth Israel Deaconess Medical Center, Boston, Massachusetts 02215
}

\begin{abstract}
The acquisition of declarative (i.e., facts) and procedural (i.e., skills) memories may be supported by independent systems. This same organization may exist, after memory acquisition, when memories are processed off-line during consolidation. Alternatively, memory consolidation may be supported by interactive systems. This latter interactive organization predicts interference between declarative and procedural memories. Here, we show that procedural consolidation, expressed as an off-line motor skill improvement, can be blocked by declarative learning over wake, but not over a night of sleep. The extent of the blockade on procedural consolidation was correlated to participants' declarative word recall. Similarly, in another experiment, the reciprocal relationship was found: declarative consolidation was blocked by procedural learning over wake, but not over a night of sleep. The decrease in declarative recall was correlated to participants' procedural learning. These results challenge the concept of fixed independent memory systems; instead, they suggest a dynamic relationship, modulated by when consolidation takes place, allowing at times for a reciprocal interaction between memory systems.
\end{abstract}

Key words: sleep; declarative learning; procedural learning; consolidation; off-line; skill learning; motor learning

\section{Introduction}

Our memories are thought to be organized into separate and distinct systems: a declarative system dealing with our memories for facts, and a procedural system dealing with our memories for skills (Cohen and Squire, 1980). The independence of these memory systems has been demonstrated by declarative learning deficits being associated with only minimal procedural learning deficits; conversely, procedural learning deficits are associated with only minimal declarative learning deficits (Cohen and Squire, 1980; Nissen and Bullemer, 1987; Gabrieli et al., 1993; Willingham, 1997; Willingham et al., 2002). Whether this concept of encapsulated memory systems applies beyond initial memory acquisition into the off-line processing of memories during consolidation is unknown.

Memory consolidation can describe the stabilization of a memory, as it becomes less susceptible to interference, or the enhancement of a memory, with task performance improving "off-line" between training sessions (Robertson et al., 2004a; Walker, 2005). Both of these expressions of consolidation have been examined using an interference technique in which the consolidation of a primary task is disrupted by immediately performing a secondary task. For example, a newly acquired skill can be lost when an individual immediately attempts to acquire skill in another distinct task (Brashers-Krug et al., 1996; Walker et al., 2003). When the time between the primary and secondary task is lengthened, the interference between the tasks is lessened. With

\footnotetext{
Received June 20, 2007; revised July 24, 2007; accepted Aug. 9, 2007.

This work was supported by National Institutes of Health Grant R01 NS051446 (E.M.R.)

Correspondence should be addressed to Edwin M. Robertson, Center for Noninvasive Brain Stimulation, Beth Israel Deaconess Medical Center, 330 Brookline Avenue, Kirstein Building KS 446, Boston, MA 02215. E-mail: emrobert@bidmc.harvard.edu.

D0I:10.1523/JNEUROSCI.2799-07.2007

Copyright $\odot 2007$ Society for Neuroscience $\quad$ 0270-6474/07/2710468-08\$15.00/0
}

less interference from the secondary task, the primary task can be consolidated. In previous studies, the primary and secondary tasks have either both required the acquisition of a procedural memory or both required the acquisition of a declarative memory. Thus, interference during consolidation has always been examined within the same memory system (i.e., procedural or declarative).

We modified those previous experimental designs, which had examined the interference within a memory system, to allow us to examine the interference between the declarative and procedural systems when consolidation took place over wake or over a night of sleep (Brashers-Krug et al., 1996; Goedert and Willingham, 2002; Walker et al., 2003). For a motor learning task we used the serial reaction-time task (SRTT) (Nissen and Bullemer, 1987), which expresses the benefits of consolidation as an off-line improvement (Robertson et al., 2004b), whereas for a declarative learning task, we used a word-list learning task, which expresses the benefits of consolidation as maintenance of word recall resulting from stabilization (Ellenbogen et al., 2006). In the first experiment, motor skill learning (i.e., SRTT) was followed by declarative learning (i.e., word-list learning), providing an assessment of how declarative learning influences motor skill consolidation. In the second experiment, declarative learning preceded motor skill learning, allowing us to examine the influence of motor skill learning on declarative consolidation. In both experiments, consolidation was measured by comparing participants' initial performance on the primary task against their later performance at retest on the same task after an interval of being awake or a night of sleep (see Fig. 1).

\section{Materials and Methods}

Participants. One hundred and twenty-five right-hand-dominant [defined by the Edinburgh handedness questionnaire (Oldfield, 1971)] par- 
ticipants were recruited. Off-line improvements, a measure of procedural consolidation, can be modified by a participant's ability to recall segments of the sequence: only those reporting four or less items consistently show improvements over wake (Robertson et al., 2004b). Data was analyzed from the remaining 92 participants (41 male; $21.1 \pm 0.3$ years) who were randomly distributed across the groups. All participants completed a sleep questionnaire and those allocated to an overnight group also completed a sleep log (slept, $6.7 \pm 0.2 \mathrm{~h}$; mean \pm SEM).

Experimental groups. Experiment 1 was designed to examine the influence of declarative learning on procedural consolidation. Participants were initially tested (at 8:00 A.M. or 8:00 P.M.) on a procedural sequence learning task, the SRTT (see below); they then learned a list of words (word-list task; see below) and, after a $12 \mathrm{~h}$ interval over a night of sleep or wake, were retested on the SRTT (at 8:00 P.M. or 8:00 A.M., respectively). After retesting, participants' declarative knowledge for the SRTT sequence was measured using a free-recall test. In a separate group of participants, the word-list task was replaced, by a task requiring them to count the number of vowels within nonsense letter strings (vowel counting task; see below). In this group, participants were tested (8:00 A.M.) and, $12 \mathrm{~h}$ later, retested (8:00 P.M.) on the SRTT.

Experiment 2 was designed to examine the influence of procedural learning on declarative consolidation; participants initially performed the word-list learning task (at 8:00 A.M. or 8:00 P.M.), they then performed the SRTT and, after a $12 \mathrm{~h}$ interval over a night of sleep or wake, they recalled the word list. In a separate group of participants, the SRTT was replaced by a task requiring participants to make a series of random visually guided finger movements [random reaction time task (RRTT)] (see below). In this group, participants recalled the word list (8:00 A.M.) and $12 \mathrm{~h}$ later had their recall of the word list retested (8:00 P.M.). Ten participants were allocated to all three groups within both sets of experiments.

In summary, participants were initially tested on a primary task, they then performed a secondary task (session 1), and $12 \mathrm{~h}$ later, they were retested on the primary task (session 2) (see Fig. 1).

Control groups. Two control groups were associated with each set of experiments. In one control group, there was only $30 \mathrm{~min}$ between testing and retesting in the primary task. For the other control group, a $4 \mathrm{~h}$ interval was inserted between the primary and secondary tasks; with retesting in the primary task occurring $12 \mathrm{~h}$ (at 8:00 P.M.) after the initial exposure to that task. Eight participants were allocated to each of these control groups.

The SRTT. We used a modified version of the SRTT (Nissen and Bullemer, 1987; Robertson, 2007). A solid circular visual cue (diameter $20 \mathrm{~mm}$, viewed from $\sim 800 \mathrm{~mm}$ ) could appear at any one of four possible positions, designated 1-4, and arranged horizontally on a computer screen. Each of the four possible positions corresponded to one of the four buttons on a response pad (RB-410; Cedrus, San Pedro, CA) on which the participants' fingers rested. When a target appeared, participants were instructed to respond by pressing the appropriate button on the pad. If the participant made an incorrect response, the stimulus remained until the correct button was selected. The position of the visual cues played out a repeating 12-item sequence (2-3-1-4-3-2-4-1-3-4-2-1).

Session one consisted of a short training block with 15 repetitions of the sequence ( 180 trials), a longer training block with twenty-five repetitions (300 trials), and a test block with 15 repetitions (180 trials) (see Fig. 1). In experiment 1, there was a second SRTT session consisting of a single test block with 15 repetitions (180 trials) of the sequence. For all blocks, 50 random trials preceded and followed the sequential trials. Within these random trials, there were no item repeats. Each set of random trials in the training and test blocks were unique. However, the random trials were identical across all groups.

RRTT. This was identical to the SRTT in every aspect except the repeating 12 -item sequence was replaced by an equal number of random trials.

Word-list task. A single word from a list of 16 words (drawn from the California Verbal Learning Test), was presented on a computer screen for $2 \mathrm{~s}$. The word was then removed and replaced by another word also drawn form the list of 16 words. This process continued until all 16 words had been presented. The words, in order, were truck, spinach, giraffe, bookcase, onion, motorcycle, cabinet, zebra, subway, lamp, celery, cow, desk, boat, squirrel, and cabbage. The same 16 words were presented individually and in the same order five times for each participant. At the end of each of these presentations, participants were asked to recall, in any order, as many of the words as possible. They were not prompted for particular words, nor were they told those words, if any, which they had failed to recall. After the fifth recall, there was a $10 \mathrm{~min}$ interval after which a free recall test of the word list was administered. In experiment 2 , an additional free-recall test was administered $12 \mathrm{~h}$ (experimental groups), $4 \mathrm{~h}$, or $30 \mathrm{~min}$ (control groups) later.

Vowel-counting task. Participants were shown a nonsense string of letters, varying in length from three to 12 letters. Participants were asked to state the number of different vowels within a string. Each string was presented for $2 \mathrm{~s}$ before being replaced by another nonsense letter string. This continued until 16 nonsense letter strings had been presented. This was a single iteration. In all there were a total of five iterations. After the fifth iteration, there was a 10 min interval, after which 16 nonsense letter strings were presented individually for $2 \mathrm{~s}$, and participants were asked to state the number of different vowels within each string. Each of the nonsense letter strings was unique.

Data analysis. Response times were defined as the time to make a correct response. Any response time longer than 2.7 SDs (i.e., the top one percentile) from a participant's mean was removed. For the SRTT, a learning score was calculated by subtracting the average response time of the final 50 sequential trials from the average response time of the random trials that immediately followed (Nissen and Bullemer, 1987; Willingham et al., 1989). Accuracy in the SRTT is not a useful measure of skill, because even with limited experience, error rates are extremely low $(<2-$ 4\%) (Willingham et al., 1989; Cohen et al., 2005). Skill before the interval ( skill $\left._{1}\right)$ was calculated using the final test block of the first session, whereas skill after the interval ( skill $_{2}$ ) was calculated using the first and only test block of the second session (see Fig. 1). For word-list learning, initial recall $\left(\right.$ recall $\left._{1}\right)$ was the number of words participants provided unprompted $10 \mathrm{~min}$ after the five iterations of seeing and recalling each of the 16 words individually (see above, Word-list learning). This free recall test was also administered $12 \mathrm{~h}$ later, providing a retest measure of word recall $\left(\right.$ recall $\left._{2}\right)$. Change in either SRTT performance ( skill $_{2}-$ skill $_{1}$ ) or word recall $\left(\right.$ recall $_{2}-$ recall $_{1}$ ) between testing and retesting provided a measure of procedural and declarative consolidation, respectively. These measures of consolidation were compared across groups using a mixed repeated measures ANOVA. Planned paired $t$ tests were used to compare skill $_{1}$ against skill ${ }_{2}$ or recall ${ }_{1}$ against recall ${ }_{2}$ to test within groups for changes in either SRTT performance or word recall.

\section{Results}

\section{Experiment 1}

Participants performed a motor skill learning task, the SRTT, and then immediately performed a secondary task. After a $12 \mathrm{~h}$ interval of wake or a night of sleep, they were retested on the SRTT (experiment 1). The secondary task was either a declarative task in which participants learned a list of words, or a vowel counting task in which participants were asked to count the number of vowels within nonsense letter strings; this involved similar perceptual processes to word-list learning but without any declarative learning. The difference between SRTT performance at testing $\left(\right.$ skill $\left._{1}\right)$ and retesting $\left(\right.$ skill $\left._{2}\right)$ provided a measure of procedural consolidation, expressed as an off-line (i.e., a between session) skill improvement ( skill $_{2}-$ skill $_{1}$ ) (Fig. 1) (Fischer et al., 2002; Walker et al., 2002; Robertson et al., 2004a,b).

\section{Procedural consolidation and the secondary task}

There was no significant difference in skill between the groups at initial testing, before performing either secondary task (word-list learning or vowel counting, skill ${ }_{1}$; ANOVA, $F_{(1,18)}<1$; $p=$ 0.947) (Fig. 2). However, the type of secondary task did have a significant impact on the development of off-line skill improvements over wakefulness (ANOVA, $F_{(1,18)}=7.217 ; p=0.015$ ). 


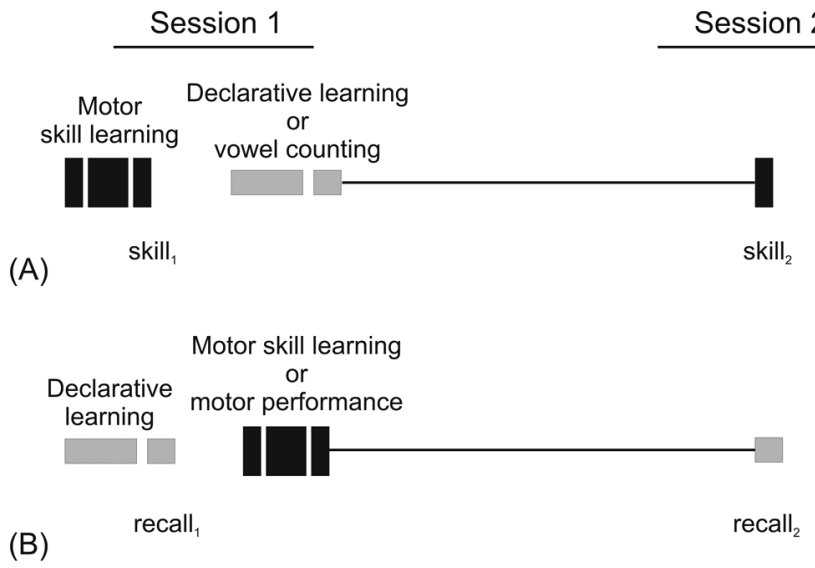

Figure 1. Experimental design. $\boldsymbol{A}$, In the first experiment, we examined the capacity of declarative learning to disrupt procedural consolidation. Participants learned a motor skill and performed a secondary task, and $12 \mathrm{~h}$ later their motor skill was retested. We contrasted the effects of two secondary tasks, word-list learning versus vowel counting, on procedural consolidation over wake, and then examined whether word-list learning had a differential capacity to interfere with procedural consolidation over wake or a night of sleep. The difference between motor skill at testing ( (kill $_{1}$ ) and retesting (skill ${ }_{2}$ ) provided a measure of procedural consolidation) (skill ${ }_{2}$ - skill $)_{1}$ ) (Walker et al., 2002; Robertson et al., 2004b; Cohen et al., 2005; Spencer et al., 2006). $\boldsymbol{B}$, In the second experiment, we examined the capacity of motor skill learning to disrupt declarative consolidation. Participants learned a list of words and performed a secondary task, and $12 \mathrm{~h}$ later their word recall was retested. We contrasted the effects of two secondary tasks, motor skill learning (i.e., SRTT) versus motor performance (i.e., RRTT), on declarative consolidation and examined whether motor skill learning had a differential capacity to interfere with declarative consolidation over wake or a night of sleep. The difference between initial (recall ${ }_{1}$ ) and subsequent recall (recall ${ }_{2}$ ) of the word list provided a measure of declarative consolidation (recall ${ }_{2}-$ recall $_{1}$ ) (Ellenbogen et al., 2006).

Off-line improvements did not develop when the secondary task required participants to learn a list of words $(-22 \pm 14 \mathrm{~ms}$; paired $t$ test, $t_{(9)}=1.589 ; p=0.146$ ) (Fig. 2). In contrast, substantial off-line improvements developed when the secondary task required participants to count the number of vowels within nonsense letter strings $\left(20 \pm 7 \mathrm{~ms}\right.$; paired $t$ test, $t_{(9)}=2.86$; $p=$ $0.019)$ (Fig. 2). These off-line improvements are similar in magnitude to those that developed when there was no intervening secondary task between SRTT testing and retesting [an average improvement of $21 \mathrm{~ms}$; data from Cohen et al. (2005)]. This implies that simply being engaged in a secondary task after motor skill learning is not sufficient to block off-line motor skill improvements. However, contrasting between these two secondary tasks cannot determine whether the blockade was specifically caused by declarative learning. To examine this issue, we correlated the disruption of procedural consolidation to the extent of declarative learning.

\section{Correlation: procedural consolidation and declarative learning}

We correlated the disruption to procedural consolidation, as measured by a change in motor skill, against declarative learning. This revealed a significant correlation with a greater decrease in motor skill being associated with a greater recall of the word list $(r=0.71 ; F=8.075 ; p=0.022)$ (Fig. 3). Such a correlation may have resulted from participants' declarative knowledge for the word list influencing the declarative component of the SRTT, and thereby altering the expression of skill (Brown and Robertson, 2007; Robertson, 2007). In such a scenario, the declarative component of the SRTT ought to have been altered by declarative knowledge for the word list but unaffected by vowel counting.
Over wake (8am to $8 \mathrm{pm})$

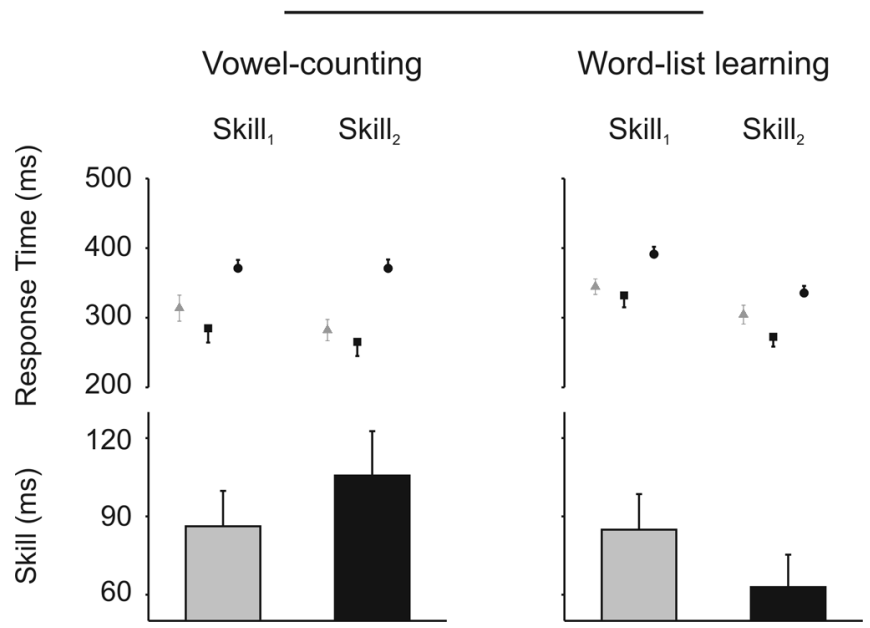

Figure 2. In experiment 1, after the acquisition of a motor skill (8:00 A.M., SRTT, skill ${ }_{1}$; gray bars \pm SEM) participants performed a secondary task (vowel counting vs word-list learning) and were retested $12 \mathrm{~h}$ later on the motor skill (8:00 P.M., skill 2 , black bars \pm SEM). Over the interval, there was a general improvement in performance: response times to the random trials (gray triangle \pm SEM) preceding the sequential trials fell significantly (vowel-counting task, paired $t$ test, $t_{(9)}=4.6, p=0.001$; word-list learning task, paired $t$ test, $t_{(9)}=4.0, p=0.003$ ). This fall in response time did not differ significantly between the groups (unpaired $t$ test, $t_{(18)}=$ $0.04 ; p=0.966)$. A similar general performance improvement was observed in the sequential (square \pm SEM) and subsequent random response times (circle \pm SEM) after word-list learning (sequential, paired $t$ test, $t_{(9)}=2.39, p=0.04$; random, paired $t$ test, paired $t$ test, $t_{(9)}=$ $6.23, p<0.001)$. Thus, with little change in the differential between the sequential and subsequent random response times, which is a widely used measure of skill in this motor task, there were no significant off-line skill improvements after word-list learning ( $-22 \pm 14 \mathrm{~ms}$; paired $t$ test, $t_{(9)}=1.589 ; p=0.146$ ) (Nissen and Bullemer, 1987; Willingham et al., 1989; Willingham and Goedert-Eschmann, 1999). In contrast, there was a decrease in sequential response times (paired $t$ test, $t_{(9)}=4.13 ; p=0.002$ ), but no significant change in the random response times (paired $t$ test, $t_{(9)}<1 ; p=0.985$ ) after vowel counting. The absence of a decrease in these latter random response times indicated the off-line development of skill ( $20 \pm 7 \mathrm{~ms}$; paired $t$ test, $t_{(9)}=2.86 ; p=0.019$ ). The development of skill encourages participants to play out the sequence even when this is inappropriate during the unexpected introduction of random trials after the sequential trials (postrandom trials). This proactive interference from the sequential onto the random trials increases as skill increases, and causes an increase in postrandom response times whereas the sequential response times decrease (Robertson, 2007). The increase in postrandom response times counteracts the general improvement in task performance, causing there to be no change in the postrandom response times. Thus, off-line skill improvements, which normally develop over wake, were present after vowel counting, but were blocked by word-list learning (Robertson et al., 2004b; Cohen et al., 2005; Press et al., 2005).

However, there was no significant difference in the declarative component of the SRTT after word-list learning compared with vowel counting $(0.9 \pm 0.5$ vs $0.6 \pm 0.4$ items from the 12 -item sequence; unpaired $t$ test, $\left.t_{(18)}<1 ; p=0.628\right)$. Furthermore, there was no correlation between participants' declarative knowledge for the word list and their declarative knowledge for the SRTT sequence $(r=0.15 ; F=0.195 ; p=0.670)$. These results both imply that word-list learning did not influence the declarative component of the SRTT. Instead, the correlation between motor skill consolidation and word-list learning was the result of a direct interaction. This correlation suggests that the disruption of procedural consolidation is dependent not simply on engaging in declarative learning, but that the extent of declarative learning is proportional to the impairment of motor skill consolidation.

\section{Procedural consolidation and the type of interval}

We also contrasted the ability of declarative learning to interfere with procedural consolidation, expressed as an off-line skill im- 


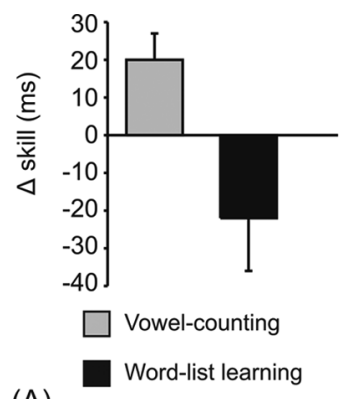

(A)

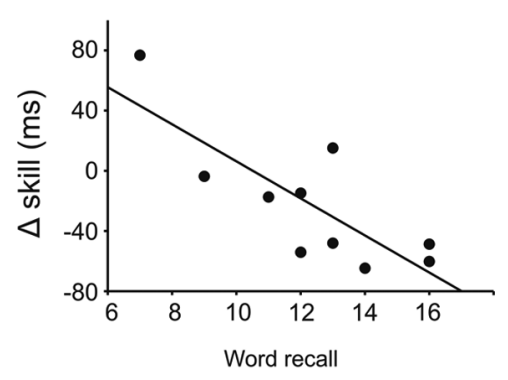

(B)

Figure 3. A, In experiment 1 , off-line skill improvements were differentially affected by the performance of another task immediately after skill acquisition: vowel counting allowed offline skill improvements to develop as normal (gray bar \pm SEM), whereas word-list learning prevented the development of significant off-line improvements (black bar \pm SEM). $\boldsymbol{B}$, The decline in motor skill after word-list learning was correlated with participants' ${ }^{\prime}$ word recall ( $r=$ $0.767 ; F=11.42 ; p=0.01$ ). In contrast, there was no relationship between the declarative component of the SRTT (free recall of the 12-item sequence) and word recall. Thus, the effect of word-list learning on motor skill performance was not mediated via an influence of word-list learning on the declarative component of the SRTT. Instead, there was a direct effect of declarative learning on procedural consolidation.
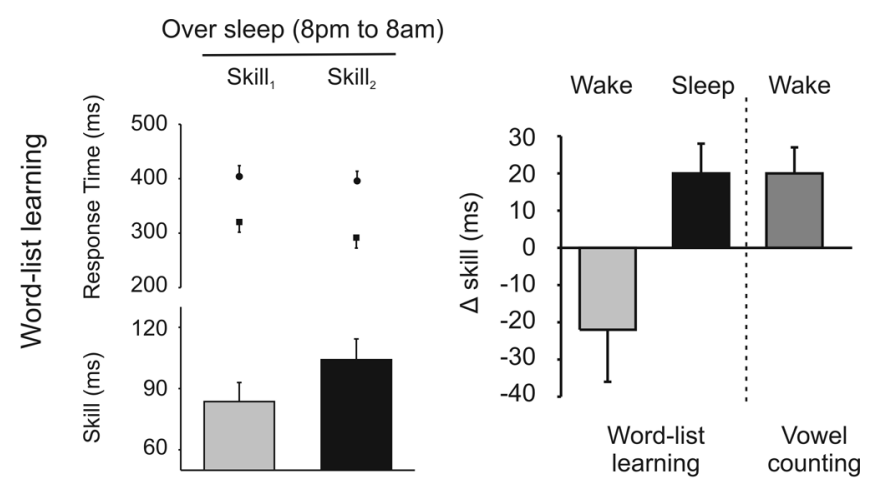

(A)

(B)

Figure 4. $\quad A$, In experiment 1 , off-line skill improvements developed over a night of sleep (8:00 P.M. to 8:00 A.M.) even although participants had earlier learned a word list ( $21 \pm 8 \mathrm{~ms}$; paired $t$ test, $\left.t_{(9)}=2.799 ; p=0.021\right)$. Producing this off-line skill improvement was a decrease in sequential response times (square $\pm \mathrm{SEM}$; paired $t$ test, $t_{(9)}=2.785 ; p=0.021$ ) combined with no significant change in the random response times (circle \pm SEM; paired $t$ test, $t_{(9)}<1$; $p=0.363) \cdot \boldsymbol{B}$, After word-list learning, off-line skill improvements were blocked over wake but developed over a night of sleep (bars \pm SEM). This differential pattern cannot be attributed to changes in the expression of skill at particular times of day. There was no significant difference in motor skill between the groups at the initial testing (8:00 A.M. vs 8:00 P.M.). Similar off-line skill improvements are expressed at 8:00 A.M. and 8:00 P.M. after a night of sleep and a day awake, respectively (Robertson et al., 2004b, 2005; Cohen et al., 2005; Spencer et al., 2006). Furthermore, off-line skill improvements can be expressed at 8:00 P.M., after an interval of wake, when participants counted vowels within nonsense letter strings immediately after motor skill learning. Thus, declarative learning has a differential capacity to block procedural consolidation over wake and a night of sleep.

provement, over both wake and a night of sleep. Regardless of the time of day at which participants were initially tested on the SRTT, we found no significant difference in performance between the groups (8:00 A.M. vs 8:00 P.M., skill ${ }_{1}$; ANOVA, $F_{(1,18)}$ $<1$; $p=0.939$ ) (Figs. 2, 4). However, the type of interval, whether it was over wake or a night of sleep, did have a significant effect on the development of off-line motor skill improvements ( skill $_{2}-$ skill $_{1}$; repeated measures, ANOVA, $\left.F_{(1,18)}=7.36 ; p=0.014\right)$. There were significant improvements over a night of sleep (21 \pm $8 \mathrm{~ms}$; paired $t$ test, $t_{(9)}=2.799 ; p=0.021$ ), whereas there were no significant improvements over wake $(-22 \pm 14 \mathrm{~ms}$; paired $t$ test, $t_{(9)}=1.589 ; p=0.146$ ) (Figs. 2, 4). Procedural consolidation was differentially disrupted despite there being no significant difference in declarative learning between the groups (unpaired $t$ test, $\left.t_{(18)}=1.49 ; p=0.153\right)$. The differential pattern can also not be attributed to the time of retesting. Off-line improvements can normally develop over both sleep and wake (Robertson et al., 2004b, 2005; Cohen et al., 2005; Spencer et al., 2006), and were detected, after a $12 \mathrm{~h}$ interval of wakefulness, at 8:00 P.M., when participants performed a vowel counting task rather than a wordlist learning task immediately after initial motor skill learning (Fig. 4). These observations demonstrate that the time of retesting (i.e., 8:00 P.M.) was not sufficient to explain the absence of off-line skill improvements over wake. Thus, word-list learning had a differential effect on procedural consolidation: blocking off-line improvements over wake, but not over a night of sleep.

\section{Control groups for experiment 1}

Learning a word list could influence performance on the SRTT by acting retroactively on information acquired during initial skill learning; alternatively, it could act proactively on SRTT performance at retesting (Goedert and Willingham, 2002). These two mechanisms can be distinguished by inserting a time interval between the initial SRTT testing and word-list learning. Inserting this interval should, according to the retroactive mechanism, decrease the influence word-list learning has on the SRTT. Consistent with this perspective, significant off-line improvements, which were absent when word-list learning took place immediately after the SRTT, were able to develop when a $4 \mathrm{~h}$ interval was inserted between word-list learning and the SRTT (17 $\pm 4 \mathrm{~ms}$; paired $t$ test, $\left.t_{(7)}=3.8 ; p=0.006\right)$. Therefore, word-list learning retroactively disrupted either the information acquired during initial SRTT training or the off-line processing of this information.

Determining whether declarative learning affects the procedural skill acquired during initial SRTT training or the off-line processing of procedural memories can be achieved by decreasing the interval between SRTT testing and retesting. This limits the opportunity for off-line processing. This manipulation should, assuming that off-line processing mediates the influence of word-list learning in the SRTT, prevent word-list learning from having an effect on SRTT performance. Consistent with this view, word-list learning did not have an immediate effect on SRTT performance: with only a 30 min interval between testing and retesting, there was no change in skill $(3 \pm 9 \mathrm{~ms}$; paired $t$ test, $\left.t_{(7)}=0.354 ; p=0.734\right)$. Thus, the disruptive effect declarative learning had on procedural consolidation was not caused by declarative learning modulating the motor skill acquired during initial SRTT training. Instead, declarative learning impaired the off-line processing of motor skill memories.

\section{Experiment 2}

In experiment 1, we examined the influence of declarative learning on procedural consolidation. We now turn to examine the reciprocal relationship: the capacity of procedural learning to interfere with declarative consolidation. This was assessed by participants first learning a declarative word list, then performing a secondary task, and, after a $12 \mathrm{~h}$ interval over wake or a night of sleep, recalling the word list (experiment 2). The secondary task was either the SRTT or a modified version of this task in which participants made a series of random visually guided finger movements: an RRTT. Both tasks required motor performance, but only one, the SRTT, involved motor sequence learning. For all the groups, the difference between word-list recall at testing 


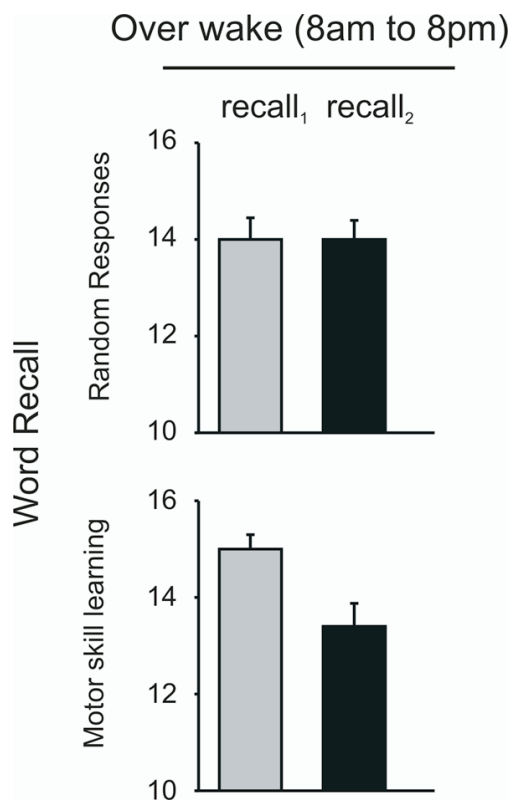

Figure 5. In experiment 2, after word-list learning (gray bars \pm SEM), participants immediately performed another task (random visually guided response vs motor skill learning), which had a differential effect on their later word recall (black bars \pm SEM). Participants' ${ }^{\prime}$ word recall was maintained over wake after they made a series of random visually guided responses $\left(0 \pm 0.4\right.$ change in word recall; paired $t$ test, $\left.t_{(9)}<0.001 ; p>0.99\right)$, whereas there was a significant decrease in word recall after participants learned a motor skill ( $-1.6 \pm 0.3$ words; paired $t$ test, $\left.t_{(9)}=5.237 ; p=0.001\right)$. Thus, declarative consolidation can be blocked by motor skill learning.

$\left(\right.$ recall $\left._{1}\right)$ and retesting $\left(\right.$ recall $\left._{2}\right)$ provided a measure of declarative consolidation $\left(\right.$ recall $_{2}-$ recall $\left._{1}\right)$ (Fig. 1).

\section{Declarative consolidation and the secondary task}

There was no significant difference in declarative recall between the groups at initial testing, before performing either of the secondary procedural tasks (SSRT or RRTT, recall ${ }_{1}$; ANOVA, $F_{(1,18)}$ $=2.63 ; p=0.123$ ) (Fig. 5). However, the type of secondary task did have a significant impact on the maintenance of declarative knowledge over wakefulness (ANOVA, $F_{(1,18)}=7.32 ; p=0.014$ ). Word recall fell significantly between initial and subsequent recall $\left(-1.6 \pm 0.3\right.$ words; paired $t$ test, $t_{(9)}=5.237 ; p=0.001$ ) (Fig. $5)$ when the secondary task involved motor skill learning (the SRTT; skill in the SRTT, $59 \pm 9 \mathrm{~ms}$; paired $t$ test, $t_{(9)}=6.6$; $p<$ $0.001)$. In contrast, when the secondary task required the performance of visually guided movements (the RRTT), word recall did not change significantly between initial and subsequent recall $\left(0 \pm 0.4\right.$ words; paired $t$ test, $\left.t_{(9)}<0.001 ; p>0.99\right)$ (Fig. 5$)$. When performing the RRTT, participants showed no significant improvement in performance: there was no significant decline in response time between the first and the final 50 trials ( $420 \pm 24$ vs $390 \pm 20 \mathrm{~ms}$; paired $t$ test, $t_{(9)}=1.87 ; p=0.1$ ). These results show that simply making a series of finger movements was not sufficient to block declarative consolidation, but instead, motor skill learning had to occur to block declarative consolidation.

\section{Correlation: declarative consolidation and motor skill learning}

We correlated the disruption to declarative consolidation, as measured by a change in word recall, against motor skill learning. This revealed a significant correlation with a greater decrease in declarative word recall being associated with greater motor skill

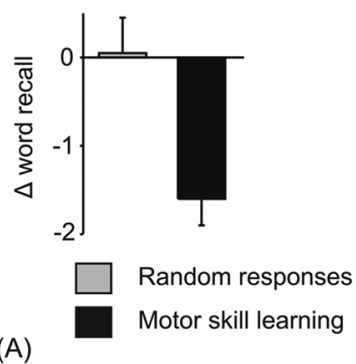

Figure 6. $\quad A$, In experiment 2 , declarative consolidation, as measured by a change in word recall between initial testing and later retesting, was differentially affected by the task performed immediately after initial word-list learning. Word recall was maintained after participants performance of a random series of visually guided movements (gray bar \pm SEM). In contrast, declarative consolidation was blocked after participants learned a motor skill (black bar \pm SEM). This disruption to declarative consolidation lead to a decrease in word recall over wake. $\boldsymbol{B}$, The decline in word recall after motor skill learning was correlated with participants' acquired skill ( $r=0.767 ; F=11.42 ; p=0.01)$. In contrast, there was no relationship between the declarative component of the SRTT (free recall of the 12-item sequence) and word recall. This implies that the declarative component of the SRTT did not influence word recall. Instead, there was a direct effect of motor skill learning on declarative consolidation.

$(r=0.767 ; F=11.42 ; p=0.01)$ (Fig. 6). The decrease in recall may have been because participants' word recall was disrupted by the declarative component of the SRTT (Robertson, 2007). However, there was only a minimal declarative component to the SRTT (1.2 \pm 0.5 items from the 12-item sequence): all those recalling, on a free-recall test, more items of the SRTT sequence than would be expected by chance were removed from the analysis (see Materials and Methods). The remaining participants had a recall for the SRTT sequence that was not significantly different from the spurious recall participants had when making random responses in the RRTT ( $1.2 \pm 0.5$ vs $1.4 \pm 0.8$; unpaired $t$ test, $t_{(18)}$ $=0.212 ; p=0.834)$. Despite both tasks having a similar declarative component, it was only those participants who learned the motor skill task (i.e., the SRTT) that showed a decrease in declarative word recall. This implies that the declarative component of the SRTT was not sufficient to cause a decrease in word recall. Furthermore, there was no correlation between the decline in word recall and participants' declarative knowledge for the SRTT sequence $(r=0.089 ; F<1 ; p=0.807)$. Thus, the significant correlation between declarative consolidation and motor skill learning appears to be the result of a direct interaction.

\section{Declarative consolidation and the type of interval}

We also contrasted the ability of motor skill learning to interfere with declarative consolidation over both wake and a night of sleep. Regardless, of the time of day at which participants learned the word list, we found no significant difference in initial recall between the groups (8:00 A.M. vs 8:00 P.M.; ANOVA, $F_{(1,18)}<1$; $p=0.722$ ). However, the type of interval, whether it was over wake or a night of sleep, had a significant effect on declarative consolidation $\left(\right.$ recall $_{2}-$ recall $_{1}$; repeated measures ANOVA, $\left.F_{(1,18)}=17 ; p=0.001\right)$. Word recall was maintained over a night of sleep $\left(0.1 \pm 0.3\right.$ words; paired $t$ test, $\left.t_{(9)}=0.361 ; p=0.726\right)$ (Fig. 7), but fell substantially over wake $(-1.6 \pm 0.3$ words; paired $t$ test, $\left.t_{(9)}=5.237 ; p=0.001\right)$. Declarative consolidation was differentially disrupted despite there being no significant difference in motor skill learning between the groups (unpaired $t$ test, $\left.t_{(18)}=0.442 ; p=0.662\right)$. The differential pattern can also not be attributed to the time of retesting. Declarative word recall was maintained after a $12 \mathrm{~h}$ interval of wakefulness, at 8:00 P.M., when participants had performed a motor task (i.e., the RRTT), 


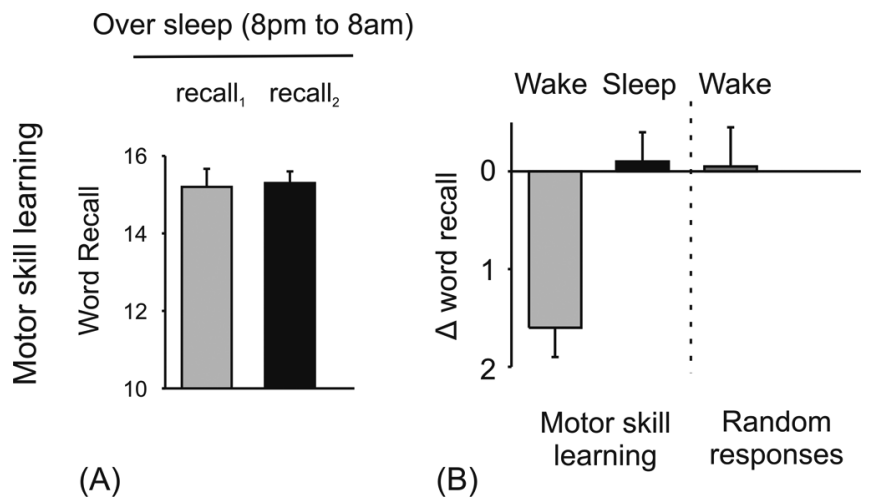

Figure 7. $\quad \boldsymbol{A}$, In experiment 2, word recall was maintained over a night of sleep despite participants having earlier learned a motor skill $\left(0.1 \pm 0.3\right.$ words; paired $t$ test, $t_{(9)}=0.361$; $p=0.726$; bars show mean \pm SEM). $\boldsymbol{B}$, Declarative consolidation, as measured by a change in word recall over wake or a night of sleep, was differentially effected by previous motor skill learning. Word recall fell over wake after motor skill learning, indicating a disruption of declarative consolidation (gray bar \pm SEM). In contrast, word recall was maintained over a night of sleep (black bar \pm SEM). This differential pattern cannot be attributed to a diurnal effect on word recall. There was no significant difference in initial recall between the groups (8:00 A.M. vs 8:00 P.M.). Furthermore, maintained recall could be expressed at 8:00 P.M., after an interval of wake, when participants made random visually guided responses immediately after initial word-list learning (dark gray bar \pm SEM). Thus, motor skill learning has a differential capacity to block declarative consolidation over wake and a night of sleep.

rather than a motor skill learning task (i.e., the SRTT) immediately after word-list learning (Fig. 7). This implies that the time of recall (i.e., 8:00 P.M.) was not sufficient to explain the decrease in word recall over wake. Thus, motor skill learning had a differential effect on declarative consolidation: causing a decrease in declarative recall over wake, but not over a night of sleep.

\section{Control groups for experiment 2}

The SRTT could influence the final recall of the word list by acting retroactively on information acquired during word-list learning; alternatively, it could act proactively on word recall at retesting (Goedert and Willingham, 2002). These two mechanisms can be distinguished by inserting a time interval between initial wordlist learning and subsequent performance on the SRTT. Inserting this interval should, according to the retroactive mechanism, decrease the influence the SRTT has on word-list recall. Consistent with this notion, instead of a decrease in word recall, which had occurred when learning the word list was immediately followed by the SRTT, there was no significant change in the number of words recalled when a $4 \mathrm{~h}$ interval was inserted between word-list learning and the SRTT $\left(-0.6 \pm 0.4\right.$ words; paired $t$ test, $t_{(7)}=1.6$; $p=0.15)$. This suggests that procedural learning acted retroactively to modulate either the declarative memory acquired during word-list learning, or the off-line processing of that declarative memory.

Determining whether procedural learning affects an acquired declarative memory or the off-line processing of that memory can be achieved by decreasing the interval between the initial and final recall of the word list. This decreases the opportunity for declarative off-line processing, which, assuming that the effect of procedural learning is mediated via an influence on off-line processing, should prevent procedural learning from having an effect on declarative word recall. Consistent with this view, there was no significant decrease, but rather a trend toward an increase in the number of words recalled when there was only a $30 \mathrm{~min}$ interval between initial and final recall $(0.88 \pm 0.6$ words; paired $t$ test, $\left.t_{(7)}=1.5 ; p=0.15\right)$. Thus, the disruptive effect of procedural learning was not caused by a modulation of the acquired declarative memory; instead, procedural learning impaired the engagement of those consolidation mechanisms responsible for maintaining the stability of the declarative memory.

\section{Discussion}

Here, we demonstrate that procedural consolidation can be disrupted by declarative learning and that the reverse is also true, with declarative consolidation being disrupted by procedural learning. Such an organization may be a feature of the processing of all, or only some, procedural and declarative memories; nonetheless, these observations demonstrate the capacity for reciprocal interactions between procedural and declarative memories. We observed these interactions over wakefulness, but not over a night of sleep. Thus, rather than there being independent memory systems, as may be the case during memory encoding, there is a reciprocal interaction between procedural and declarative memory systems during consolidation. This is not a fixed relationship; instead, it is dynamic, with the interaction between memory systems being modulated by when consolidation takes place.

As participants acquire skill at producing a sequence while performing the SRTT, they also acquire an ability to declaratively describe the sequence (Nissen and Bullemer, 1987; Willingham et al., 1989; Robertson, 2007). We minimized the influence of this component by removing from the study those individuals who were able to recall more items from the 12 -item sequence (i.e., $>3$ items) than would be expected by chance (Willingham and Goedert-Eschmann, 1999). This minimal declarative recall was no different from the recall given by participants making random responses while performing the RRTT. Thus, we minimized declarative knowledge for the SRTT and thereby minimized the propensity for this SRTT component to interact with declarative knowledge of the word list. Despite this precaution, word-list learning may have influenced the declarative component of the SRTT and thereby perhaps altered the expressed motor skill (experiment 1). This seems unlikely because there was no relationship between declarative recall for the SRTT sequence and word recall. Instead, there was a significant correlation between word recall and the decline in motor skill. This implies that there was a direct interaction between declarative learning and procedural consolidation. Equally in experiment 2, the declarative component of the SRTT may have influenced word recall. However, there was no relationship between declarative recall for the SRTT sequence and the decrease in word recall. In contrast, there was a significant correlation between the decrease in declarative recall and motor skill. This implies that there was a direct interaction between procedural learning and declarative consolidation. Thus, these patterns of correlation suggest that there were reciprocal interactions between the declarative and procedural memory systems.

The reciprocal interactions between memory systems are present over wakefulness, but not over a night of sleep. Off-line skill improvements are blocked over wake by learning a word list whereas improvements continue to develop over a night of sleep. Similarly, there is a decrease in declarative word recall over wake by learning a motor skill whereas word recall remains unchanged over a night of sleep. This pattern of results cannot be explained by diurnal factors because the time of day did not significantly influence the expression of skill or recall. Furthermore, additional groups within this study, consistent with previous work, showed that declarative and procedural consolidation can be expressed both at 8:00 P.M., after an interval of wake, or at 8:00 


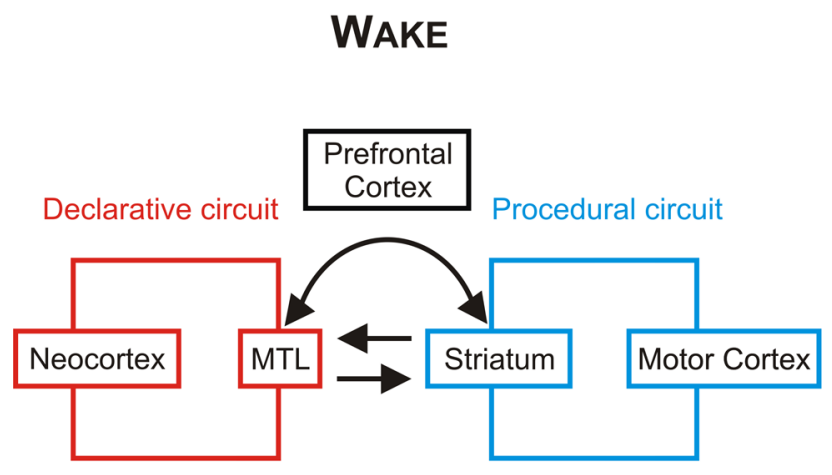

(A) Interconnected systems

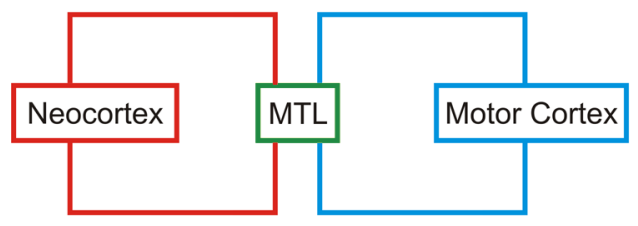

(B) Overlapping systems

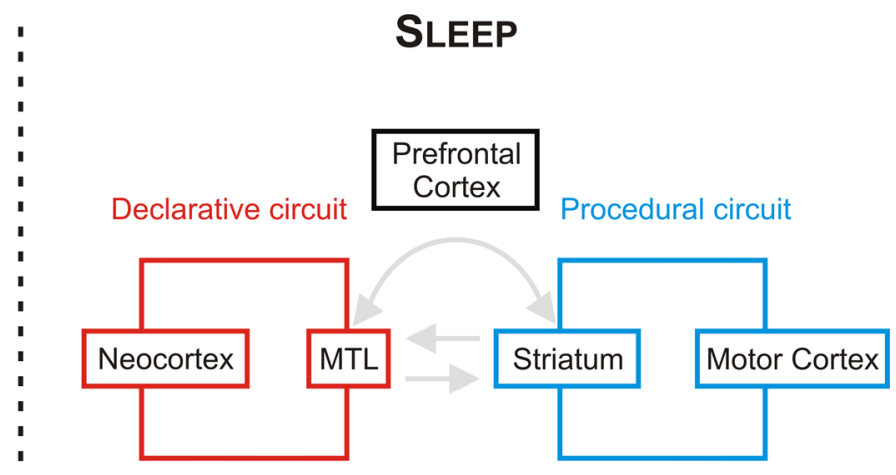

(C) Functional Uncoupling
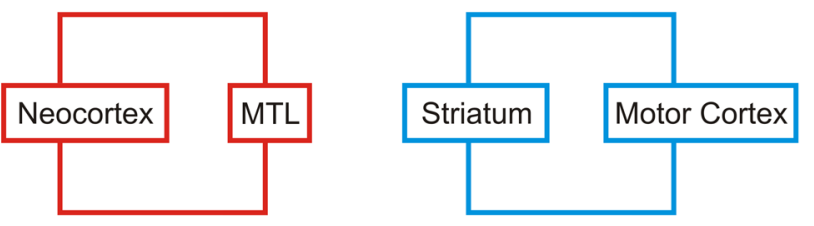

(D) Independent systems

Figure 8. The biological infrastructure that may account for our observations. $A$, Memory systems may interact over wake because of direct or indirect connections. The indirect connections may arise from brain areas, such as the prefrontal cortex, controlling or receiving processing from otherwise distinct memory systems (Poldrack et al., 2001; Voermans et al., 2004). $\boldsymbol{B}$, Alternatively, the processing of declarative and procedural memories may engage similar or at least partially overlapping neural circuits. Previous work has shown some overlap between the processing of declarative and procedural memories (Curran, 1997; Schendan et al., 2003; Walker et al., 2005). Such neuronal overlap could account for the behavioral interference between declarative and procedural processing over wake. In contrast, over sleep, memory systems cease to interact. C, Potentially, this is because of a decrease in the functional connectivity among brain areas (Massimini et al., 2005). This would allow declarative and procedural systems to operate as independent memory systems. $\boldsymbol{D}$, Alternatively, a change in brain state from wake to sleep may open up independent pathways for both procedural and declarative consolidation. This would require, as has been shown in some previous studies that differential mechanisms support consolidation over wake and sleep (Cohen et al., 2005; Robertson et al., 2005; Robertson and Cohen, 2006). The neuronal circuits engaged over wake may have the property of allowing interactions between memory systems whereas a differential set of circuits, engaged over sleep, may support independent memory processing.

A.M. after a night of sleep (Figs. 4, 7) (Robertson et al., 2004b; Cohen et al., 2005; Spencer et al., 2006).

Several biological mechanisms may allow declarative and procedural memory processing to interact over wake, but operate independently over a night of sleep. The off-line processing of declarative or procedural memories causes the engagement of specific neural circuits. These may be distinct circuits. A network, including the primary motor cortex, has been shown to support procedural consolidation whereas a circuit including the medial temporal lobe (MTL), hippocampus, and neocortex may support declarative consolidation (Robertson et al., 2005; Peigneux et al., 2006; Takashima et al., 2006; Rasch et al., 2007). Despite being distinct circuits, interactions between the processing of declarative and procedural memories, like those described by our results, could arise either from direct connections between the different procedural and declarative circuits or by indirect connections mediated by other brain areas (Voermans et al., 2004) (Fig. 8). Alternatively, the circuits processing declarative or procedural memories may include overlapping regions. Previous work has shown that activation of the MTL is associated with processing procedural or declarative memories (Curran, 1997; Schendan et al., 2003; Walker et al., 2005). Thus, the interference between memory systems may arise because they share common neuronal resources or because of direct or indirect connections between the memory systems (Fig. 8).

The absence of an interaction between declarative and procedural processing over a night of sleep may result from any connections between memory systems ceasing to operate. This could be achieved, for either direct or indirect connections, by changes in functional connectivity. As the brain shifts from wake to sleep there are dramatic neurochemical and neurophysiological changes, which can produce a decrease in functional connectivity among brain areas (Hobson and Pace-Schott, 2002; Massimini et al., 2005). Thus, sleep may functionally disconnect declarative and procedural systems, allowing them to operate as independent memory systems. Alternatively, the shift from wake to sleep may open up new mechanisms capable of supporting the consolidation of procedural and declarative memories. Distinct mechanisms appear to be engaged to support motor skill consolidation over wake and sleep (Cohen et al., 2005; Robertson et al., 2005). These alternative routes, opened by sleep, may allow declarative and procedural processing that no longer shares common neuronal resources. This would produce independent memory systems operating free of reciprocal interactions. Thus, a functional disconnection or the emergence of nonoverlapping circuits may explain the absence of an interaction between procedural and declarative processing over sleep (Fig. 8). Future studies will perhaps be able to distinguish among these possibilities.

In summary, the off-line processing of a procedural memory can be blocked by declarative learning, and the off-line processing of a declarative memory can be blocked by procedural learning. This reciprocal interaction between memory systems is not a fixed relationship; instead, the extent of the interaction or independence between memory systems is modulated by when consolidation takes place. Thus, rather than a fixed organization of two independent encapsulated memory systems operating in parallel, procedural and declarative memory systems show a flexible capacity to, at times, remain independent, while at other times interact in a reciprocal manner to support human performance. 


\section{References}

Brashers-Krug T, Shadmehr R, Bizzi E (1996) Consolidation in human motor memory. Nature 382:252.

Brown RM, Robertson EM (2007) Inducing motor skill improvements with a declarative task. Nat Neurosci 10:148-149.

Cohen DA, Pascual-Leone A, Press DZ, Robertson EM (2005) Off-line learning of motor skill memory: A double dissociation of goal and movement. Proc Natl Acad Sci USA 0506072102.

Cohen NJ, Squire LR (1980) Preserved learning and retention of patternanalyzing skill in amnesia: dissociation of knowing how and knowing that. Science 210:207-210.

Curran T (1997) Higher order associative learning in amnesia: evidence from the serial reaction time task. J Cogn Neurosci 9:522-533.

Ellenbogen JM, Hulbert JC, Stickgold R, Dinges DF, Thompson-Schill SL (2006) Interfering with theories of sleep and memory: sleep, declarative memory, and associative interference. Curr Biol 16:1290-1294.

Fischer S, Hallschmid M, Elsner AL, Born J (2002) Sleep forms memory for finger skills. Proc Natl Acad Sci USA 99:11987-11991.

Gabrieli JD, Corkin S, Mickel SF, Growdon JH (1993) Intact acquisition and long-term retention of mirror-tracing skill in Alzheimer's disease and in global amnesia. Behav Neurosci 107:899-910.

Goedert K, Willingham D (2002) Patterns of interference in sequence learning and prism adaptation inconsistent with the consolidation hypothesis. Learn Mem 9:279-292.

Hobson J, Pace-Schott E (2002) The cognitive neuroscience of sleep: neuronal systems, consciousness and learning. Nat Rev Neurosci 3:679-693.

Massimini M, Ferrarelli F, Huber R, Esser SK, Singh H, Tononi G (2005) Breakdown of cortical effective connectivity during sleep. Science 309:2228-2232.

Nissen MJ, Bullemer P (1987) Attentional requirements of learning: evidence from performance measures. Cognit Psychol 19:1-32.

Oldfield R (1971) The assessment and analysis of handedness: the Edinburgh inventory. Neuropsychologia 9:97-113.

Peigneux P, Orban P, Balteau E, Degueldre C, Luxen A, Laureys S, Maquet P (2006) Offline persistence of memory-related cerebral activity during active wakefulness. PLoS Biol 4:e100.

Poldrack RA, Clark J, Pare-Blagoev EJ, Shohamy D, Creso Moyano J, Myers C, Gluck MA (2001) Interactive memory systems in the human brain. Nature 414:546-550.

Press DZ, Casement MD, Pascual-Leone A, Robertson EM (2005) The time course of off-line motor sequence learning. Cognitive Brain Res 25:375-378.

Rasch B, Buchel C, Gais S, Born J (2007) Odor cues during slow-wave sleep prompt declarative memory consolidation. Science 315:1426-1429.
Robertson EM (2007) The serial reaction time task: implicit motor skill learning? J Neurosci 27:10073-10075.

Robertson EM, Cohen DA (2006) Understanding consolidation through the architecture of memories. Neuroscientist 12:261-271.

Robertson EM, Pascual-Leone A, Miall RC (2004a) Current concepts in procedural consolidation. Nat Rev Neurosci 5:576-582.

Robertson EM, Pascual-Leone A, Press DZ (2004b) Awareness modifies the skill-learning benefits of sleep. Curr Biol 14:208-212.

Robertson EM, Press DZ, Pascual-Leone A (2005) Off-line learning and the primary motor cortex. J Neurosci 25:6372-6378.

Schendan HE, Searl MM, Melrose RJ, Stern CE (2003) An FMRI study of the role of the medial temporal lobe in implicit and explicit sequence learning. Neuron 37:1013-1025.

Spencer RM, Sunm M, Ivry RB (2006) Sleep-dependent consolidation of contextual learning. Curr Biol 16:1001-1005.

Takashima A, Petersson KM, Rutters F, Tendolkar I, Jensen O, Zwarts MJ, McNaughton BL, Fernandez G (2006) Declarative memory consolidation in humans: a prospective functional magnetic resonance imaging study. Proc Natl Acad Sci USA 103:756-761.

Voermans NC, Petersson KM, Daudey L, Weber B, Van Spaendonck KP, Kremer HP, Fernandez G (2004) Interaction between the human hippocampus and the caudate nucleus during route recognition. Neuron 43:427-435.

Walker MP (2005) A refined model of sleep and the time course of memory formation. Behav Brain Sci 28:51-64.

Walker MP, Brakefield T, Morgan A, Hobson JA, Stickgold R (2002) Practice with sleep makes perfect: sleep-dependent motor skill learning. Neuron 35:205-211.

Walker MP, Brakefield T, Hobson JA, Stickgold R (2003) Dissociable stages of human memory consolidation and reconsolidation. Nature 425:616-620.

Walker MP, Stickgold R, Alsop D, Gaab N, Schlaug G (2005) Sleepdependent motor memory plasticity in the human brain. Neuroscience 133:911-917.

Willingham DB (1997) Systems of memory in the human brain. Neuron 18:5-8.

Willingham DB, Goedert-Eschmann K (1999) The relation between implicit and explicit learning: Evidence for parallel development. Psychol Sci 10:531-534.

Willingham DB, Nissen MJ, Bullemer P (1989) On the development of procedural knowledge. J Exp Psychol Learn Mem Cogn 15:1047-1060.

Willingham DB, Salidis J, Gabrieli JD (2002) Direct comparison of neural systems mediating conscious and unconscious skill learning. J Neurophysiol 88:1451-1460. 